\title{
Health Warning Label Compliance for Smokeless Tobacco Products and Bidis in Five Indian States
}

\author{
Sejal Saraf ${ }^{1 *}$, Kevin Welding ${ }^{1}$, Michael Iacobelli', Joanna E Cohen², Prakash C \\ Gupta $^{3}$, Katherine C Smith ${ }^{1}$
}

\begin{abstract}
Background: The burden of tobacco use In India is very high. To inform users of harm, India has a strong health warning label law that applies to all tobacco products. This study examines the extent of compliance of health warning labels on smokeless tobacco (SLT) and bidi products with the Indian law. Methods: In 2017, a systematic protocol was used to collect unique SLT and bidi packages from five Indian states. To assess compliance, we used three indicators: location, label elements, and warning size. Results: Only 1\% of the 133 SLT products and none of the 32 bidi packs were compliant with all three compliance indicators. Other compliance-related issues included non-standardized packaging, incomplete health warning labels, poor printing quality, and old warning labels. Conclusion: There is very poor compliance with the health warning label law on bidi and SLT products. India needs to regularly monitor and address implementation to ensure that warning labels are effective.
\end{abstract}

Keywords: Smokeless tobacco- bidis- health warning label- compliance- tobacco- packaging- India

Asian Pac J Cancer Prev, 22, Progress of Tobacco Control in the South-East Asia Region Suppl, 59-64

\section{Introduction}

India is the world's second leading consumer, the third largest producer and the fifth largest exporter of tobacco products (Mishra et al., 2012). According to the Global Adult Tobacco Survey (GATS) survey (2016-17), $10.7 \%$ (99.5 million) of the Indian adult population smoked tobacco. Bidis (processed tobacco flakes, hand-rolled in a tendu leaf) were the most commonly used smoked product: $7.7 \%$ smoked bidis while $4.0 \%$ smoked cigarettes. Smokeless tobacco products (SLT) are even more prevalent; these products were used by $21.4 \%$ (199.4 million) and, of note, a higher proportion of women used SLT than combustible products. Given the extent of SLT and bidi use in India, and their significant health harms (Gupta et al., 2003; Critchley et al., 2003; Jha et al., 2008; Jitender et al., 2008; Gupta et al., 2016) effectively communicating the harmful effects of these products is imperative.

The World Health Organization's (WHO) Framework Convention on Tobacco Control (FCTC) has identified graphic health warnings labels (HWLs) on product packaging as a cost-effective policy intervention to inform consumers about tobacco's health risks. Graphic warnings can impact people with low levels of literacy (WHO
Framework Convention on Tobacco Control, 2003). Given SLT and bidi consumers are more likely to have little or no formal schooling, graphic HWLs are particularly useful in these settings (Sorensen et al., 2005). Aligning with the FCTC guidelines, the government of India has made substantial strides in strengthening graphic HWLs. Most recently, the Cigarettes and Other Tobacco Products Act (COTPA) set HWL requirements to cover $85 \%$ of the principal display on both sides of all tobacco packages (Ministry of Health and Family Welfare Notification G.S.R 182(E), 2008; Ministry of Health and Family Welfare Notification G.S.R 182(E), 2014).

The efficacy of a HWL is influenced by several collective elements such as the size, position, content, and message design (Fong et al., 2009; Swayampakala et al., 2015). Also, to be effective in achieving its ultimate health goals, the HWL needs to be implemented as intended (Cohen et al., 2016).

Few studies have investigated HWL compliance with regard to COTPA, particularly in rural settings. One study conducted in four urban cities in India found HWL compliance rates of $51 \%$ for cigarettes but only $2 \%$ for SLT products (Tobacco Pack Surveillance System, 2016). Another very small study conducted in the rural areas of Bhubaneswar found that none of the bidi packages carried 
a compliant HWL with appropriate location and coverage (Panigrahi and Sharma, 2019). A third study that reviewed Indian tobacco policies noted that those that covered SLT products were either inadequate or poorly implemented (Khan et al., 2014).

In India, SLT and bidi consumers primarily reside in rural settings, and most of the product brands are local and vary by region. To date, the few studies that have assessed HWL compliance in India have mostly been conducted in urban settings, with relatively small sample size. Given the burden and pattern of tobacco use in India, rigorous compliance assessment studies are crucial to understand the current status of implementation of packaging laws. This study aimed to assess the compliance of health warning labels on SLT and bidi products in semi-urban and rural areas across five states in India, and to identify possible barriers to implementation.

\section{Materials and Methods}

In October and November 2017, a systematic protocol was used to purchase all unique SLT and bidi packages available from a random sample of vendors within five states in India: Maharashtra, Uttar Pradesh, Rajasthan, Karnataka, and Assam. These states were selected based on geographic diversity, prevalence of SLT users, and political relevance (i.e., states with political will for policy change). Within each state, the top five most populous districts were shortlisted for data collection. From these five districts, we pragmatically selected two to three districts, based on geographic proximity. Using the Census Bureau of India (CBI) tiers (Census of India, 2011), within each selected district we identified one semi-urban town with population of $20,000-49,999$ (tier 3), two rural villages with populations of 10,000 - 19,999 (tier 4), and two rural villages with populations of 5,000-9,999 (tier 5) [Appendix-1].

In each town or village, vendors were selected using a walking protocol that originated from a point of interest or "hub" that was identified from a compiled census of religious temples, educational institutions, and postal offices. The primary source for hubs was Google Maps, with additional information gathered from local municipal websites. Up to five, but no less than three geographically distinct hubs were pre-selected before data collection began. Where available, multiple backup hubs were identified in the event that a primary hub was incorrect or missing. Within each identified town or village and using the hubs identified, the walking protocol was used to construct a random sample of tobacco vendors from which unique SLT and bidi packages were purchased.

Individual vendors selected were chosen randomly, but the types of vendors selected for sampling was based on the most popular tobacco vendor types in India according to Euromonitor, GATS, and guidance from local partners; the tobacco vendor types were small grocery stores, paan bidi shops, street vendors, and tobacco specialists [Appendix-2]. Data collectors initiated the walking protocol from a hub and implemented the walking protocol to find one of the four vendor types.
Upon encountering a tobacco vendor, data collectors systematically assessed all available tobacco products. Unique packs were defined as packs that had at least one difference in an exterior feature of the pack. Any pack with a different design or feature, including packs with differing tobacco quantities, brand presentation, and colors was considered unique. Packs that were exactly the same except for different iterations of the country's health warning label were not considered to be distinct. At the first store in each town or village, the protocol required the purchase of one of every unique tobacco package. In subsequent hubs, we purchased any unique packs available that we had not yet purchased. In total, we purchased unique packs from 125 vendors across five states: 96 small grocery stores, 22 paan bidi shops, five street vendors, and two tobacco specialists.

To assess compliance of each package, we developed a codebook based on India's HWL requirements. Two coders independently applied the codebook to assess compliance of each pack that had at least one product appropriate, current rotation HWL. Discrepancies between coders were resolved by a third coder. We applied three indicators of HWL compliance based on a review of key elements of the Indian law: (1) HWL location - positioned on the top edge of the package on the front and back principal display areas (diametrically opposite for cylinders); (2) HWL elements - complete, free of distortion (i.e., a clear well-defined HWL), 4-color graphic HWL, warning text printed in a single language on each side, "WARNING " in white font color on a red background, product-specific warning text in white font color on a black background; and (3) HWL size $-85 \%$ of the visible principal display area covered by the HWL with $60 \%$ covered by the HWL graphic and $25 \%$ covered by the HWL warning text, and a minimum of $3.5 \mathrm{~cm}$ wide and $4 \mathrm{~cm}$ tall. At the time of data collection, the required HWL image for SLT products was the frontal view of a male face with mouth cancer on the right side, and for bidis it was the frontal view of a male neck with throat cancer [Appendix-3]. For compliance assessment, packages that had at least one in-rotation product-specific HWL (as specified for the last 12 months of the 2016-18 cycle) were included in the sample and evaluated.

\section{Results}

In total, 240 state-unique SLT packages and 71 state-unique bidi packages were collected from the five states: Maharashtra-SLT: 30; bidi: 8; Uttar Pradesh-SLT: 90, bidi: 15; Rajasthan-SLT: 42; bidi: 21; Karnataka-SLT: 36, bidi: 19; Assam-SLT: 42; bidi: 8. Table 1 presents the descriptive characteristics of the bidi and SLT packs.

\section{Health warning label compliance}

Fifty-five percent (133/240) SLT packages and $45 \%(32 / 71)$ bidi packages had at least one appropriate HWL. Of the 107 excluded SLT packs, 68 packs had an out-of-rotation HWL graphic (specified for the first 12 months of the 2016-18 cycle), 35 packs had an older Indian HWL, and four packs either had no HWL or had a foreign HWL. Of the excluded 39 bidi packs, 26 had an out-of-rotation HWL and 13 packs had an older HWL. 

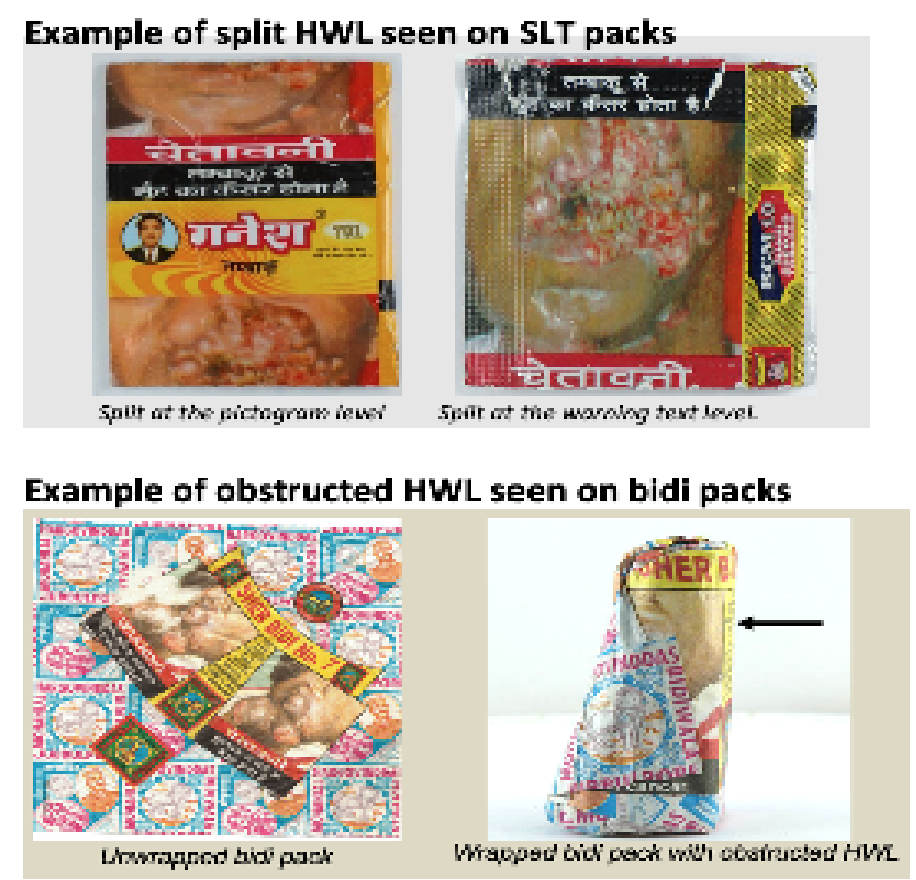

Figure 1. Packs with Incomplete HWL

\section{Compliance by indicator}

Health warning location: $36 \%(n=48)$ SLT packs and no bidi packs were compliant with the warning location indicator. Compliance varied by pack shape - for SLT, $39 \%(n=46)$ of the 119 non-cylindrical packs were compliant with location, whereas only $14 \%(n=2)$ of cylindrical SLT packs had HWLs located at the top edge and diametrically opposite to each other. Similarly, among the 29 cone-shape/cylindrical bidi packs, although seven packs had HWLs positioned diametrically opposite to each other and three packs had the HWL located at the top (widest) edge of the pack, none fulfilled both requirements

Table 1. Descriptive Characteristics of the Bidi and SLT Packages

\begin{tabular}{lccc}
\hline & SLT $(\mathrm{N}=133)$ & \multicolumn{2}{c}{ Bidi $(\mathrm{N}=32)$} \\
\hline Sachet & $115(86 \%)$ & Cone & $29(91 \%)$ \\
Cylinder & $14(10 \%)$ & Rectangle & $3(8 \%)$ \\
Hard pack & $2(2 \%)$ & & \\
Other* & $2(2 \%)$ & Pack material \\
& & & $30(94 \%)$ \\
Foil & $113(85 \%)$ & paper & $2(6 \%)$ \\
Paper & $9(7 \%)$ & plastic & \\
Tin & $7(5 \%)$ & & \\
Cardboard & $2(2 \%)$ & & \\
Plastic & $2(2 \%)$ & & \\
& & Pack weight/Stick count \\
$<10$ grams & $40(30 \%)$ & $<10$ sticks & $2(6 \%)$ \\
$>=10$ grams & $40(30 \%)$ & $10-20$ sticks & $10(31 \%)$ \\
Not listed & $53(40 \%)$ & $>20$ sticks & $20(63 \%)$ \\
\hline
\end{tabular}

* Other SLT pack shapes included (1) re-sealable pouch and (1) pouch with flap. simultaneously (Table 2).

Health warning label elements: $25 \%(n=33)$ of the SLT packs and $6 \%(\mathrm{n}=2)$ of the bidi packs were compliant with all components of the label elements indicator. Most SLT and bidi packs fulfilled the language and full color graphic specifications; however, there was significant variation in the printing quality of the graphic and completeness of the image. Among the 100 SLT packs that did not meet the label elements requirement, 91 packs had some sort of image distortion and 32 packs had a split/incomplete HWL. Similarly, among the 31 non-compliant bidi packs, $71 \%(n=22)$ had distorted HWL images and 23\% $(n=7)$ had HWLs obstructed by product wrapping which resulted in incomplete HWLs.

\section{Health warning size}

Packs were least compliant for HWL size. Only 2\% $(n=2)$ of SLT packs and one bidi pack had compliant labels (overall coverage of $85 \%$, appropriate text (25\%) and graphic $(60 \%)$ coverages and the required minimum dimensions). One SLT pack had a label that covered $85 \%$ of the package but did not have the correct text-graphic proportions. In terms of text-graphic ratio, 11 SLT packs were compliant with the $25 \%$ text size coverage but not compliant with the $60 \%$ graphic size coverage, of which two packs had a graphic coverage of less than $40 \%$. Seventy percent of SLT packs $(n=93)$ and $25 \%(n=8)$ of bidi packs had HWLs that satisfied the minimum height $(4 \mathrm{~cm})$ and width $(3.5 \mathrm{~cm})$ requirement. Most packs (62\% SLT, 28\% bidi packs) had HWL coverage between $50-69.99 \%$.

\section{Overall compliance}

One of the 133 SLT packs $(0.8 \%)$ and none of the 32 bidi packs $(0 \%)$ was compliant with all three compliance indicators. 


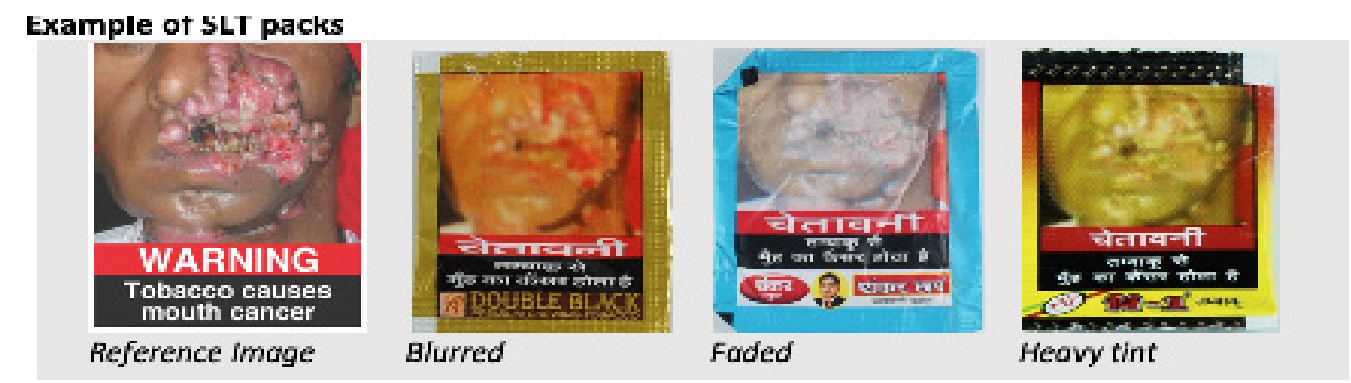

\section{Example of bidi packs}
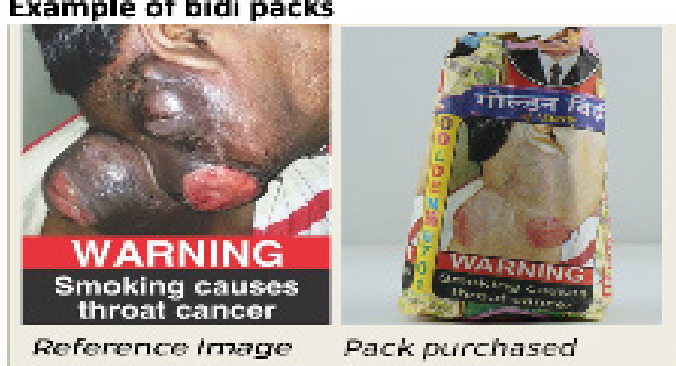

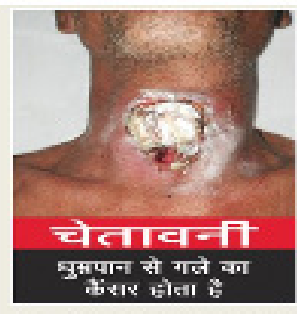

Reference smage

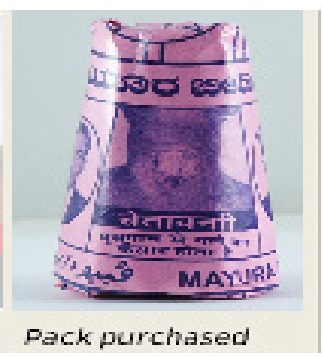

Figure 2. Printing Variation in Graphic

\section{Additional compliance-related issues}

Non-standard pack shape and size: There were five different shapes of SLT packs: sachet -115 , cylinder -14 , hard pack-2, pouch-2; bidi packs had two different shapes: cone -29 and rectangle -3 . There were significant differences in pack sizes. For SLT, the non-cylindrical pack height ranged from 4.6 to $17.8 \mathrm{~cm}$ and the width ranged from 2.9 to $12.4 \mathrm{~cm}$; the cylindrical pack height ranged from 1.8 to $10 \mathrm{~cm}$ and circumferences ranged from 5.6 to $20.4 \mathrm{~cm}$. We found that nine packs (7\%) were too small to even hold a compliant HWL, i.e., $3.5 \mathrm{~cm}$ wide ( $7 \mathrm{~cm}$ circumference) and $4 \mathrm{~cm}$ tall. For bidis, the cone-shape pack circumference of the larger end ranged from 7.9 to $13.8 \mathrm{~cm}$.

Incomplete health warning labels (split/obstructed graphics): Among the 133 SLT packs, 24\% $(n=32)$ had split HWLs: 19 had the HWL split through the pictogram and 13 had the HWL split through the text. The HWLs on bidi packs were incomplete due to obstruction by the wrapping material; $23 \%(n=7)$ packs had one or more obstructed HWLs (Figure 1).

Inconsistency in the HWL printing quality: Based on observation, 91 SLT packs and 22 bidi packs had distorted HWL images that were either blurry, faded or had heavy tint, making the HWL appear unclear. For the 91 distorted image SLT packs, 79 had HWLs with entirely blurred images, eight had selectively blurred images, and 13 had either heavy tint, faded colors, and/or stretching. Among the 22 distorted image bidi packs, six had completely blurred or selectively blurred HWLs, and 16 had either heavy tint, faded colors, or stretching (Figure 2).

\section{Outdated HWL}

Another area of concern was the large number of packs that had either out-of-rotation or previous cycle HWLs. The Indian law specifies that packs with an expired HWL shall not be distributed or sold. In our sample of 240 SLT packs, $28 \%(n=68)$ of packs had an out-of-rotation HWL graphic specified for the first 12 months of the 2016-18 cycle. Among the 32 bidi packs, 55\% $(n=39)$ had an out-of-rotation HWL(Figure 3).

\section{Discussion}

Findings from this study indicate there is overall poor compliance of HWLs on tobacco packs, with only one SLT pack $(0.8 \%)$ and none of the bidi packs being fully compliant with all three indicators. Given that India has one of the strongest packaging and labeling laws in the South-East Asia region with a requirement of $85 \% \mathrm{HWL}$ coverage, these extremely low compliance numbers indicate a need for enhanced enforcement efforts and



Figure 3. Out of Rotation Graphic HWL Images 
implementation. In order to optimize the impact of these HWLs, the government needs to ensure that manufacturers are printing the HWLs on the packs following all the requirements specified in the law.

Each of the three indicators (location, label elements, size) had poor compliance, but the $85 \%$ overall coverage component of the size indicator fared the worst. Most packs in this sample had HWL coverage that was only 50 $70 \%$. This is particularly concerning when studies show that larger health warning labels are more effective in encouraging smokers to quit and preventing nonsmokers from starting to smoke (Hammond et al., 2006; Hammond et al., 2007; Gravely et al., 2016).

Packaging dimensions and shape varied widely across the sample for both SLT and bidi packs. According to the GATS (2016-17), as compared to cigarettes which have standard pack shape and dimensions, the percentage of users who noticed HWLs on packages were lower for smokeless tobacco and bidi. In our sample, there were some SLT packs that were too small to even hold a HWL that met the minimum dimensions requirement. The bidi packages in our sample were mostly hand-wrapped in conical paper packs that have curved surfaces; these curvatures distort the image, preventing exposure to all elements of the warning label in a way that is impactful. The lack of standard shape and size stands as a challenge to the required application of the HWLs. Future research could recommend a standard design that would strengthen the salience and impact of these warnings.

The issues of incomplete and distorted HWLs are also important to note. The incomplete, split or obstructed HWLs seen on 32 SLT packs and 17 bidi packs are problematic. With regard to the split HWLs on the SLT packs, this perhaps is a product of faulty printing by the smaller local manufacturers that make up the India SLT market. The obstructed HWLs seen on bidi packs are due to the inconsistent hand-wrapping packaging technique that results in overlapping edge that cover the HWLs on the pack. Further, eight SLT packs and five bidi packs showed signs of intentional manipulation of the HWL by selectively blurring portions of the HWL graphic. There were 11 SLT packs in the sample that were compliant with the text size coverage but non-compliant with the graphic size coverage, of which two packs had a pictogram coverage of less than $40 \%$ [Appendix-4]. This implies that manufacturers are manipulating the text-to-graphic ratios of the HWLs, reducing the prominence of the graphic. There needs to be monitoring of these manufacturers on a regular basis to ensure the printing quality of the graphics are maintained.

The presence of outdated HWLs indicated intentional or unintentional errors by the vendor or manufacturer. We found $45 \%(n=170)$ of the 240 state-unique SLT packs and $55 \%(\mathrm{n}=39)$ of the 71 state-unique bidi packs for sale by vendors had the previous cycle Indian HWL, and even older versions of the Indian HWL, foreign HWLs, or no HWL. Despite the Indian law having outlined specific HWLs for each 12-month cycle, it is evident that this is not the only HWL found on shelves. For a small portion of the SLT sample (five packs), the HWL included was for a smoked tobacco product, which could mean the 
implementation guidelines are unclear or not being followed closely by manufacturers. This is concerning when evidence suggests that the impact of health warnings and messages that are repeated tends to decrease over time (Hitchman et al., 2014) Adopting HWLs that align well with the FCTC guidelines is an effective strategy for increasing awareness of the harmful effects of tobacco use, but only if well implemented.

There are limitations of this study. The current study sample is from semi-urban and rural towns in five Indian states; the landscape of products and their manner of production may be different in other regions, so the data may not reflect country-wide compliance. The data collection strategy produced unique SLT and bidi packs within the five states so the same pack could be purchased in more than one state. The $85 \%$ coverage of HWLs were measured on unopened wrapped bidi packs; the coverage might be different if HWLs were measured on unwrapped packages. Although a systematic protocol was used and the packs were double coded, the assessment of the printing quality of HWLs was subjective. Further research could assess HWL printing quality by objectively measuring dots per inch (DPI) with the appropriate equipment.

Despite the limitations, a key strength of this study is the large number of packs that were collected and assessed for compliance across 125 semi-urban and rural neighborhoods. This study helps bring these products into the spotlight and finds very poor compliance across all five states studied. The heterogeneity seen in the packaging size, the manipulation and poor printing quality of the graphics, and the manufacturing flaws are not helping SLT and bidi users to see the health warnings they need.

\section{Author Contribution Statement}

None.

\section{Acknowledgements}

We acknowledge and thank Healis Sekhsaria Institute for Public Health who collected and coded the packs under the overall supervision of Dr. Namrata Puntambekar.

The publishing cost was provided by WHO office in South-East Asia.

\section{References}

Cohen JE, Brown J, Washington C, et al (2016). Do cigarette health warning labels comply with requirements: a 14-country study. Prev Med, 93, 128-34.

Critchley JA, Unal B (2003). Health effects associated with smokeless tobacco: a systematic review.

Fong GT, Hammond D, Hitchman SC (2009). The impact of graphic pictures on the effectiveness of tobacco health warnings. Bull World Health Organ, 87, 640-3.

Gravely S, Fong GT, Driezen P, et al (2016). An examination of the effectiveness of health warning labels on smokeless tobacco products in four states in India: findings from the TCP India cohort survey. BMC Public Health, 16, 12-46.

Gupta PC, Arora M, Sinha D, Asma S, Parascondola M (2016). Smokeless tobacco and public health in India: executive summary.
Gupta PC, Ray CS (2003). Smokeless tobacco and health in India and South Asia. Respirology, 8, 419-31.

Hammond D, Fong GT, Borland R, et al (2007). Text and graphic warnings on cigarette packages: findings from the International Tobacco Control Four Country study. Am J Prev Med, 32, 202-9.

Hammond D, Fong GT, McNeill A, Borland R, Cummings KM (2006). Effectiveness of cigarette warning labels in informing smokers about the risks of smoking: findings from the International Tobacco Control (ITC) Four Country Survey. Tob Control, 15, 19-25.

Hitchman SC, Driezen P, Logel C, Hammond D, Fong GT (2014). Changes in effectiveness of cigarette health warnings over time in Canada and the United States, 2002-2011. Nicotine Tob Res, 16, 536-43.

Jha P, Jacob B, Gajalakshmi V, et al (2008). A nationally representative case-control study of smoking and death in India. N Engl J Med, 358, 1137-47.

Jitender S, Sarika G, Sharma P, Mishra P (2017). Bidi smoking: an underestimated issue of Indian Society. J Exp Ther Oncol, $12,73$.

Khan A, Huque R, Shah S, et al (2014). Smokeless tobacco control policies in South Asia: a gap analysis and recommendations. Nicotine Tob Res, 16, 890-4.

Ministry of Health and Family Welfare Notification G.S.R 182(E). March 15, 2008 [Internet]. Available from: https:// www.tobaccocontrollaws.org/files/live/India/India\%20\%20 G.S.R.\%20182\%28E\%29.pdf.

Ministry of Health and Family Welfare Notification G.S.R 182(E). October 15, 2014 [Internet]. Available from: https:// www.tobaccocontrollaws.org/files/live/India/India\%20-\%20 G.S.R.\%20727\%28E\%29.pdf.

Mishra G, Pimple S, Shastri S (2012). An overview of the tobacco problem in India. Indian J Med Paediatr Oncol, 33, 139-45.

Panigrahi A, Sharma D (2019). Compliance with packaging and labelling rules for tobacco products marketed in slum areas of Bhubaneswar, India. Tob Control, 28, e13-5.

Rural urban distribution of population: census of India. 2011 [Internet]. Available from: http://censusindia.gov.in/2011prov-results/paper2/data_files/india/Rural_Urban_2011.pdf.

Sorensen G, Gupta PC, Pednekar MS (2005). Social disparities in tobacco use in Mumbai, India: the roles of occupation, education, and gender. Am J Public Health, 95, 1003-8.

Swayampakala K, Thrasher JF, Hammond D, et al (2019). Pictorial health warning label content and smokers' understanding of smoking-related risks - a cross-country comparison. Health Educ Res, 30, 35-45.

Tata Institute of Social Sciences (TISS) (2003). Mumbai and Ministry of Health and Family Welfare, Government of India. Global Adult Tobacco Survey (GATS) India. 2016-17. Thorax, 58, 435-43.

Tobacco Pack Surveillance System (TPackSS). Health warning label compliance: India-2016 [Internet]. Available from: https://globaltobaccocontrol.org/tpackss/sites/default/files/ tpackss_indiawave2_healthwarning_06_28_18.pdf.

World Health Organization guidelines for implementation of article 11 of the WHO Framework Convention on Tobacco Control (packaging and labelling of tobacco products). 2003. This work is licensed under a Creative Commons Attribution-
Non Commercial 4.0 International License. 